\title{
Assisted reproductive technology represents a possible risk factor for development of epimutation-mediated imprinting disorders for mothers aged $\geq$ 30 years
}

Kaori Hara-Isono ${ }^{1,2}$, Keiko Matsubara ${ }^{1}$, Masashi Mikami ${ }^{3}$, Takahiro Arima ${ }^{4}$, Tsutomu Ogata ${ }^{1,5}$, Maki Fukami ${ }^{1}$ and Masayo Kagami ${ }^{1 *}$ (D)

\begin{abstract}
Backgrounds: The proportion of assisted reproductive technology (ART)-conceived livebirths of patients with imprinting disorders (IDs) is higher than that of the general population. Whether this is due to ART or confounding effects of advanced parental age was not investigated. We examined the association of ART and parental ages at childbirth for the development of eight epimutation-mediated imprinting disorders (epi-IDs).

Results: We enrolled 136 patients with epi-IDs and obtained general population ART data from the Japanese robust nationwide registry. We compared the proportion of ART-conceived livebirths and maternal childbearing ages between patients with epi-IDs and the general population. The proportion of ART-conceived livebirths in patients with epi-IDs was higher than that in mothers aged $\geq 30$ years, the age group in which more than $90 \%$ of ART procedures performed. The maternal childbearing ages of patients with epi-IDs were widely distributed from 19 to 45 (median: 32) within the approximate 2.5th to 97.5th percentiles of maternal childbearing ages of the general population. In addition, we compared the proportion of ART-conceived livebirths and parental ages at childbirth across patients with eight epi-IDs. We demonstrated that more than $90 \%$ of ART-conceived patients with epi-IDs were found in Silver-Russell syndrome (SRS) and Beckwith-Wiedemann syndrome (BWS) patients, and parental ages were almost consistent in patients with eight epi-IDs, except Prader-Willi syndrome.

Conclusions: According to the prerequisite that most of the ART procedures in Japan are performed on mothers aged $\geq 30$ years, ART can be a risk factor for the development of epi-IDs, particularly SRS and BWS, for mothers aged $\geq 30$ years.
\end{abstract}

Keywords: Assisted reproductive technology, Imprinting disorders, Epimutation, Maternal age, Risk factors

\footnotetext{
*Correspondence: kagami-ms@ncchd.go.jp

'Department of Molecular Endocrinology, National Research Institute for

Child Health and Development, 2-10-1 Okura, Setagaya-ku, Tokyo 157-8535,

Japan

Full list of author information is available at the end of the article
}

C C The Author(s). 2020 Open Access This article is licensed under a Creative Commons Attribution 4.0 International License, which permits use, sharing, adaptation, distribution and reproduction in any medium or format, as long as you give appropriate credit to the original author(s) and the source, provide a link to the Creative Commons licence, and indicate if changes were made. The images or other third party material in this article are included in the article's Creative Commons licence, unless indicated otherwise in a credit line to the material. If material is not included in the article's Creative Commons licence and your intended use is not permitted by statutory regulation or exceeds the permitted use, you will need to obtain permission directly from the copyright holder. To view a copy of this licence, visit http://creativecommons.org/licenses/by/4.0/. The Creative Commons Public Domain Dedication waiver (http://creativecommons.org/publicdomain/zero/1.0/) applies to the data made available in this article, unless otherwise stated in a credit line to the data. 


\section{Background}

Assisted reproductive technology (ART) is widespread in developed countries [1]. In Japan, 54,110 livebirths, accounting for one in 18.1 neonates, were born using ART in 2016 [2]. Artificial manipulations are possible to alter epigenetic modification of gametes and embryos. In fact, several studies revealed the impact of ART procedures on methylation imprints [3, 4]. Genomic imprinting is a marking mechanism to identify parental origin for the mono-allelic expression of imprinted genes. Differentially methylated regions (DMRs) in the imprinted regions function as the imprinting control center [5]. DNA methylation at the DMRs is a critical epigenetic modification for the regulation of expression of the imprinted genes [5]. DNA methylation imprints are erased in primordial germ cells and re-established in a sex-specific manner during gametogenesis [6]. After fertilization, although global demethylation occurs, DNA methylation imprints in embryos are maintained. ART procedures may affect this genomic imprinting in gametogenesis and embryonic development in the preimplantation stage in several ways. Controlled ovarian stimulation (COS) and in vitro maturation (IVM) of oocytes can interfere with acquisition of maternal genomic imprinting during oogenesis. In fact, superovulated human oocytes showed aberrant methylation levels of the H19DMR and MEST-DMR [7], and IVM-derived oocytes also showed aberrant methylation levels of the KCNQ1OT1-DMR [8]. In vitro fertilization (IVF), intracytoplasmic sperm injection (ICSI), and embryo culture can interfere with the maintenance of genomic imprinting in the early embryos. More than $50 \%$ of cultured preimplantation day three embryos and blastocysts showed aberrant methylation levels of the H19-, SNRP $N$-, and KCNQ1OT1-DMRs, demonstrating that ARTderived embryos possessed a high frequency of imprinted methylation errors [9]. In addition, cord blood and placentas from pregnancies conceived by IVF and ICSI had higher methylation levels of the PLAGL1-DMR [10] and lower methylation levels of the H19-DMR and MEST-DMR [11], respectively, compared with those from pregnancies conceived by natural conception. These studies suggested that IVF and ICSI altered the epigenetic signatures of offspring. However, it remains unclear whether ART procedures directly affect methylation imprints, or whether the parental issues concerned with ART, such as infertility and advanced parental age, lead to abnormal methylation. Indeed, impairment of sperm DNA methylation in male infertility and the correlation between parental advanced age at childbirth and levels of DNA methylation in the offspring have been reported $[12,13]$.

Imprinting disorders (IDs) are clinical syndromes associated with disruption of imprinted gene expression [6].
The etiologies of IDs are pathogenic variants in causative genes, copy number variants (CNVs) involving the imprinted regions, uniparental disomy (UPD) of chromosomes having imprinted genes, and epigenetic changes of the disease-responsible DMRs, i.e., epimutation. The aberrant methylation at the paternally methylated germline-derived DMRs causes Silver-Russell syndrome (SRS), Beckwith-Wiedemann syndrome (BWS), Kagami-Ogata syndrome (KOS), and Temple syndrome (TS14). On the other hand, aberrant methylation at the maternally methylated germline-derived DMRs causes BWS, Prader-Willi syndrome (PWS), Angelman syndrome (AS), pseudohypoparathyroidism 1B (PHP1B), and transient neonatal diabetes mellitus (TNDM). Previous epidemiological studies showed that the proportion of pregnancies conceived by ART was higher in patients with SRS, BWS, PWS, and AS than in the general population $[14,15]$. Previous reports have some limitations, as follows: (1) there was no investigation for the associations between ART and the remaining four IDs such as KOS, TS14, PHP1B, and TNDM; (2) there was no study which focused only on epimutation-mediated imprinting disorders (epi-IDs); (3) the confounding effect of advanced parental age at childbirth remains to be elucidated. To clarify whether ART or the confounding effect of parental age at child birth facilitates the development of epi-IDs, we examined (1) the proportion of ART-conceived livebirths and the distribution of maternal childbearing ages in patients with epi-IDs and the general population for each year and (2) the proportion of ART-conceived livebirths and parental ages at childbirth across patients with eight epi-IDs.

\section{Results}

\section{Numbers of the patients with epi-IDs}

We enrolled 136 patients with epi-IDs confirmed by molecular studies as described in methods and obtained their clinical information about parental age, conception (naturally or ART-conceived), and ART methods utilized in ART-conceived patients. According to the definition used in the Japan Society of Obstetrics and Gynecology (JSOG) database, we classified patients conceived with IVF, ICSI, and frozen embryo transfer (FET) as ARTconceived livebirths and patients born after COS only were not included in ART-conceived livebirths. The numbers of the patients with eight epi-IDs and affected DMRs are shown in Table 1. Of note, we included 31 BWS patients consisting of nine patients with the hypermethylated H19/IGF2:IG-DMR (BWS subgroup-1) and 22 patients with the hypomethylated KCNQ1OT1:TSSDMR (BWS subgroup-2). Of 136 patients with epi-IDs, information about paternal age and maternal age at childbirth were obtained from 131 and 134 patients, respectively. 
Table 1 The numbers of the patients with eight representative epi-IDs and affected DMRs

\begin{tabular}{lll}
\hline epi-IDs & Affected DMRS & $n$ \\
\hline Silver-Russell syndrome & H19/IGF2:IG-DMR hypomethylation & 77 \\
Beckwith-Wiedemann syndrome & H19/IGF2:IG-DMR hypermethylation & 9 \\
& KCNQ1OT1:TSS-DMR hypomethylation & 22 \\
Kagami-Ogata syndrome & MEG3/DLK1:IG-DMR and MEG3:TSS-DMR hypermethylation \\
Temple syndrome & MEG3/DLK1:IG-DMR and MEG3:TSS-DMR hypomethylation \\
Prader-Willi syndrome & SNURF:TSS-DMR hypermethylation \\
Angelman syndrome & SNURF:TSS-DMR hypomethylation \\
Pseudohypoparathyroidism 1B & GNAS AVB:TSS-DMR hypomethylation \\
Transient neonatal diabetes mellitus & PLAGL1:alt-TSS-DMR hypomethylation \\
Total & & 4
\end{tabular}

epi-IDs epimutation-mediated imprinting disorders, DMRs differentially methylated regions

\section{Characteristics of birth data of the Japanese general population}

We combined the data obtained from the Annual Nationwide Survey Data from the Ministry of Health, Labor (MHLW) and those from the JSOG and revealed the trends of ART pregnancy and maternal childbearing age of the Japanese general population. The approximate median and 2.5th to 97.5th percentiles of maternal childbearing age were calculated using MHLW database as described in methods. Figure 1 shows the distribution of maternal childbearing ages of ART pregnancy and that of non-ART pregnancy in Japan between 2007 and 2017. Maternal childbearing ages in both groups had become higher year by year. Maternal childbearing age of the most common group in ART pregnancy (35-39 years old) was higher than that in non-ART pregnancy

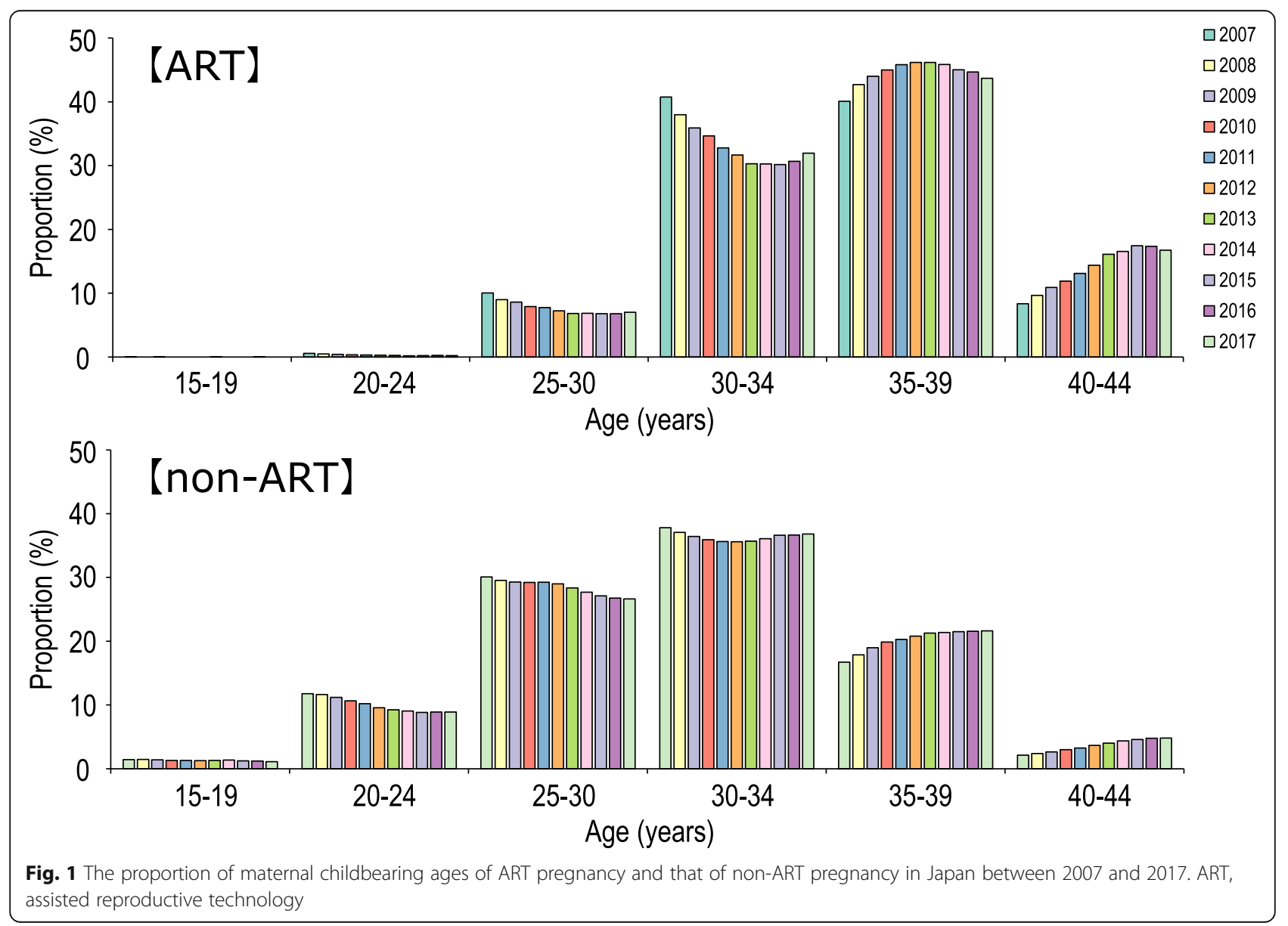


(30-34 years old). In ART pregnancy, the proportion of mothers aged $\geq 30$ years was more than $90 \%$ and that of mothers aged $<30$ years was only $7 \%$. On the other hand, the proportion of mothers aged $\geq 30$ years was about $60 \%$ in non-ART pregnancy.

\section{Comparison of the proportion of ART-conceived livebirths and maternal childbearing ages between patients with epi-IDs and the general population}

To clarify whether ART and advanced childbearing age bear the risk for the development of epi-IDs, we compared (1) the proportion of ART-conceived livebirths and (2) the distribution of the maternal childbearing ages, between patients with epi-IDs and the general population. Of 136 patients with epi-IDs, 22 patients (16.2\%) were conceived with ART. In ART-conceived patients, 12, six, and four patients were born from IVF, ICSI, and FET, respectively. Figure $2 \mathrm{a}$ shows the comparison of the proportion of ART-conceived livebirths in the general population and that in patients with epi-IDs every year from 1992 to 2017. The proportion of ARTconceived livebirths was higher in the patients with epiIDs than that in the general population, particularly from 2004 to 2017 (Fig. 2a). Because the proportion of mothers aged $\geq 30$ years was more than $90 \%$ in ART pregnancy, we compared the proportion of ARTconceived livebirths in all patients with epi-IDs and that in the general population of childbearing age $\geq 30$ years from 2007 to 2017 when we could obtain the age distribution of mothers who conceived with ART from the JSOG database. Patients with epi-IDs showed a high proportion of ART-conceived livebirths when compared to the general population of maternal age $\geq 30$ years (Fig. $2 \mathrm{~b})$. Figure 3 shows the distribution of maternal childbearing ages in the patients with epi-IDs between 1992 and 2017. The approximate median maternal childbearing age of the general population was 27 years old until 2002, then up to 32 years old in 2003 and beyond. The maternal childbearing ages of the patients with epi-IDs varied widely from 19 to 45 with the median age of 32 . In addition, most of the maternal childbearing ages of the patients with epi-IDs were distributed within the approximate 2.5 th to 97.5 th percentiles of maternal childbearing ages of the general population.

\section{Comparison of the proportion of ART-conceived livebirths and parental ages at childbirth across patients with eight epi-IDs}

To investigate the effect of ART and parental age for the development of epi-IDs in detail, we compared the proportion of ART-conceived livebirths and parental ages at childbirth across patients with eight epi-IDs. Figure 4 shows the number of ART-conceived livebirths and the distribution of parental ages at childbirth in patients with eight epi-IDs. ART-conceived livebirths were identified in patients with SRS (15.6\%), BWS (25.8\%; subgroup-1 [22.2\%], subgroup-2 [27.3\%]), TS14 (25.0\%), and PHP1B (12.5\%). Twenty out of 22 (90.9\%) ARTconceived livebirths in epi-IDs were patients with SRS or BWS. On the contrary, ART-conceived livebirth was not found in KOS, PWS, AS, and TNDM. Both the medians of paternal and maternal age at childbirth were consistent in patients with eight epi-IDs, except PWS (Fig. 4b). Notably, in PWS patients, the distributions of paternal (range 38-45) and maternal ages (range 37-39) at childbirth were higher than those in the other epi-IDs, although only four PWS patients were included in this study.

\section{Discussion}

This study is the first trial to evaluate the association of ART and parental age at childbirth for the development of IDs focusing on epimutation. We included 136 patients with epi-IDs, the largest study population enrolled so far, and performed cross-sectional analysis of eight representative epi-IDs, for the first time. Furthermore, we utilized the robust data from a nationwide ART registry system, which was characterized by its mandatory reporting system and high compliance rate [2].

Comparison between patients with epi-IDs and the general population revealed several notable findings. First, we found a higher prevalence of ART-conceived livebirths in patients with epi-IDs than in the general population (Fig. 2a). In Japan, more than $90 \%$ of ARTconceived livebirths were born from mothers aged $\geq 30$ years (Fig. 1). Therefore, when we consider the impacts of ART for the development of epi-IDs, we can investigate its effects only for mothers aged $\geq 30$ years. According to this prerequisite, we compared the proportion of ART-conceived livebirth of patients with epi-IDs to that of the general population of maternal childbearing age $\geq$ 30 years (Fig. 2b). Patients with epi-IDs including the mothers of all ages showed a high proportion of ARTconceived livebirths when compared to the general population of maternal childbearing age $\geq 30$ years. Thus, we demonstrate that ART performed on mothers aged $\geq 30$ is likely to facilitate the epi-IDs. Second, the maternal childbearing ages of patients with epi-IDs were widely distributed within the 2.5th to 97.5th percentiles of that of the general population (Fig. 3). Conversely, a previous study about UPD-mediated PWS (maternal UPD of chromosome 15) revealed that the distribution of maternal childbearing ages was significantly skewed to the advanced ages and implied that the advanced childbearing age was a predisposing factor for the development of UPD because of increased meiotic errors [16]. Unlike UPD-mediated IDs, there seemed to be no association between the development of epi-IDs and 
$1 / 1$
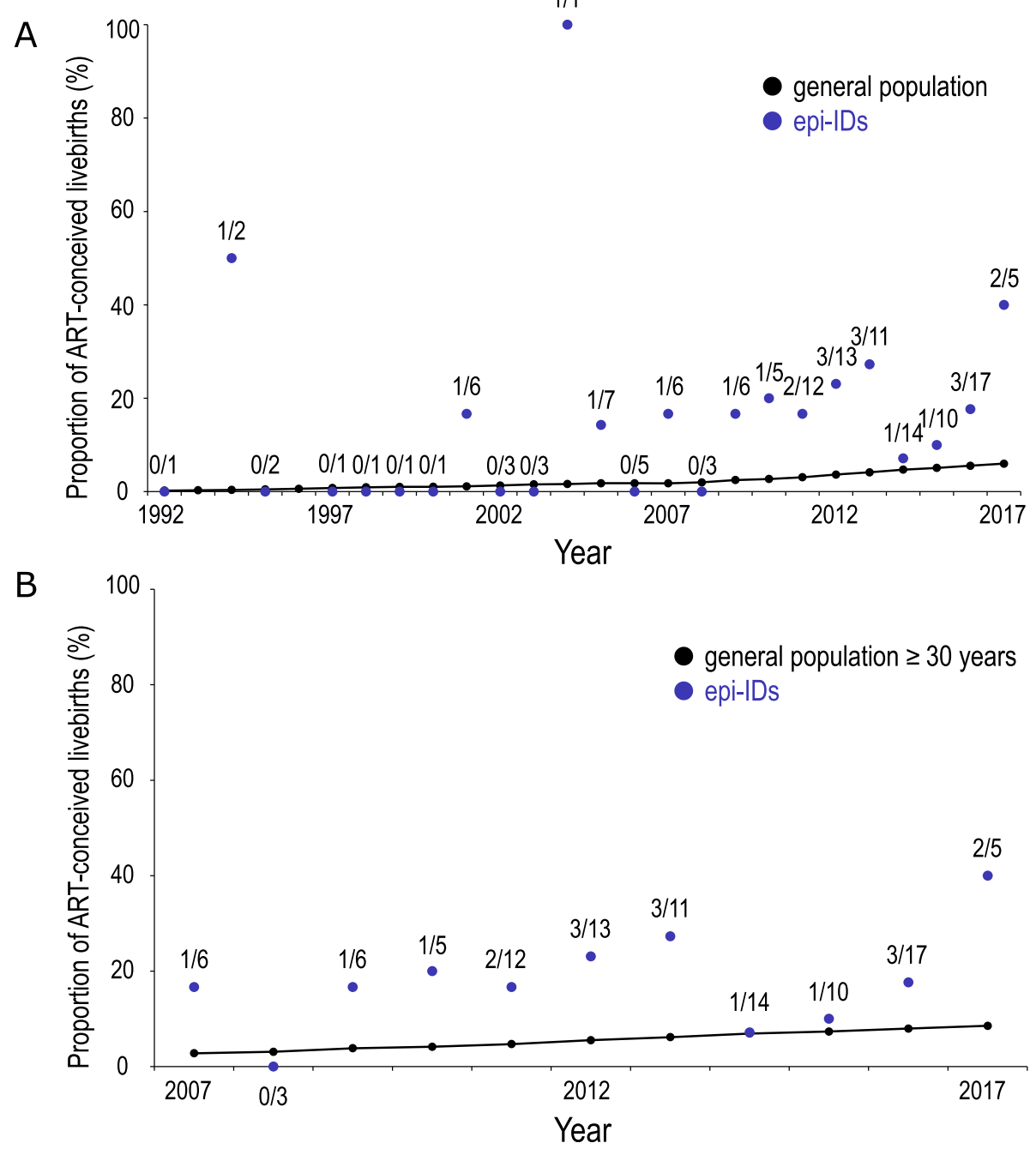

Fig. 2 The proportion of ART-conceived livebirths in the general population and that in patients with epi-IDs. Blue dots indicate the proportion of ART-conceived livebirths in patients with epi-IDs with actual numbers. a Between 1992 and 2017. Black dots represent the proportion of ARTconceived livebirths in the general population. b Between 2007 and 2017. Black dots indicate the proportion of ART-conceived livebirths in the general population of maternal age $\geq 30$ years. ART, assisted reproductive technology; epi-IDs, epimutation-mediated imprinting disorders

maternal childbearing age. This fact is compatible with a recent study in which advanced maternal age had no effect on imprinted methylation acquisition in mouse oocytes and postzygotic imprinted methylation maintenance in mouse embryos [17].

Several matters should be pointed out regarding comparison of the proportion of ART-conceived livebirths and parental ages across patients with eight representative epi-IDs. First, we compared our results to the previous studies investigating the proportions of ARTconceived livebirths in IDs (Table 2) [14, 18-21]. Only our study included all eight representative IDs focusing on epimutations and performed molecular analyses in all patients. The proportions of ART-conceived livebirths in epimutation-mediated SRS (15.6\%) and BWS (25.8\%) in our study were higher than the previously reported proportion of ART-conceived livebirths in SRS and BWS including all genetic causes (Table 2). We speculate that the methylation status of SRS and BWS related DMRs (H19/IGF2:IG-DMR, KCNQ1OT1:TSS-DMR) is particularly vulnerable to the effects of ART. This finding raises the possibility that epimutations of SRS and BWS related DMRs are more susceptible to the effect of ART than other genetic etiologies, such as UPD of chromosomes 7 and 11. In our study, approximately $90 \%$ of ARTconceived livebirths in epi-IDs were found in SRS and BWS, whereas previous studies showed that about 30 to $50 \%$ of ART-conceived livebirths in IDs were found in SRS and/or BWS (Table 2). This result is due to the fact that epimutation is the most common genetic cause of SRS and BWS (Table S1) and previous studies included patients with genetic causes other than epimutations 


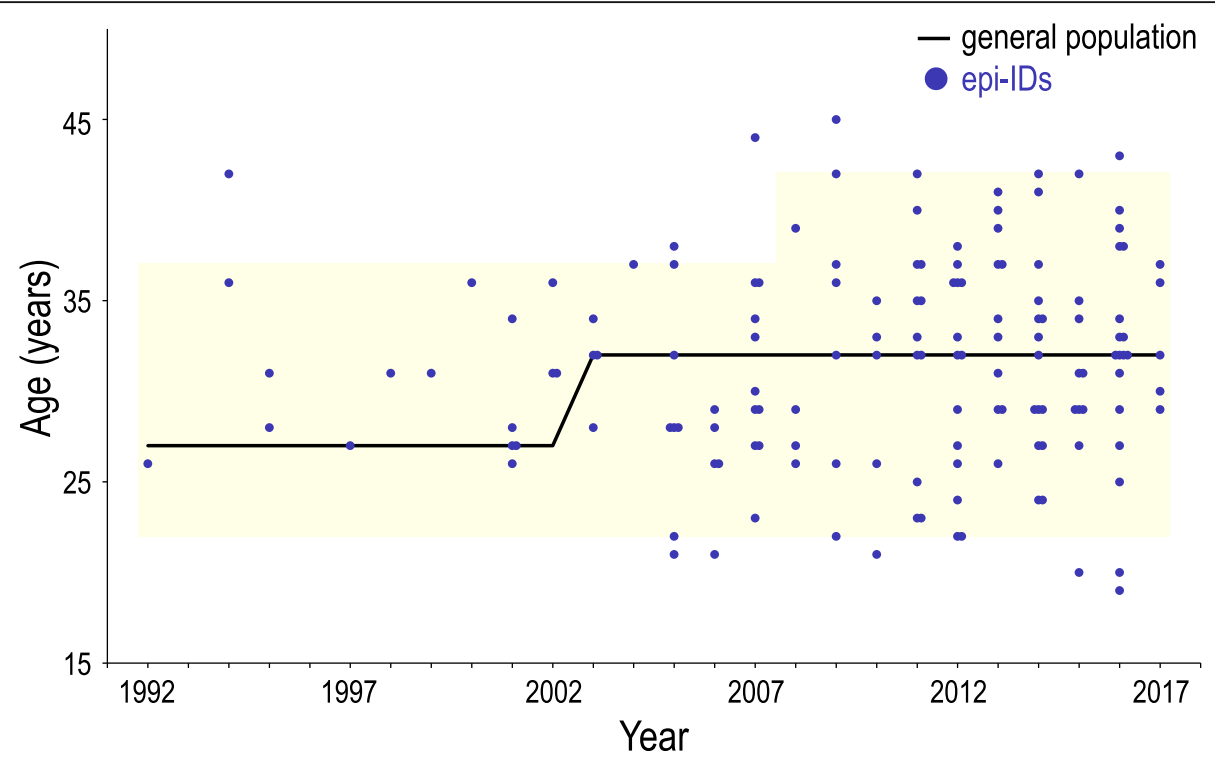

Fig. 3 The distribution of maternal childbearing ages in the patients with epi-IDs between 1992 and 2017. Blue dots indicate the maternal ages of the patients with epi-IDs in each year. The black line indicates the median age of the general population and the yellow area indicates the 2.5th to 97.5 th percentiles of the general population. epi-IDs, epimutation-mediated imprinting disorders

resulting in IDs. Indeed, patients with SRS or BWS accounted for approximately $80 \%$ of the patients included in our study. Unlike previous studies, we found no ARTconceived livebirths in epimutation-mediated PWS and AS patients (Table 2). This could be explained by the rarity of epimutation in PWS $(<1 \%)$ and AS $(<3 \%)$ patients. Second, we evaluated the proportion of ART-conceived livebirths in KOS, TS14, PHP1B, and TNDM and found two ART-conceived livebirths, one in TS14 and one in PHP1B. We previously reported two ART-conceived livebirths with TS14, one in epimutation and one in UPD [22]. Regarding PHP1B, only two livebirths following ART had been reported and all of them were caused by the loss of methylation at GNAS $A / B$ locus (epimutation) [23, 24]. These results might reflect the association between ART and epimutation, particularly in TS14 and PHP1B. Third, parental age at childbirth was consistent in eight epi-IDs except PWS. This may be explained by the assumption that parental age at childbirth has a small or no effect on the specific DMRs. In fact, a previous study in fetal cord blood of healthy infants revealed that parental age at childbirth has almost no effect on the methylation levels of the DMRs [25]. Additionally, both paternal and maternal age at childbirth in PWS patients were higher than those in the other epi-IDs. Because we included a limited number of epimutation-mediated PWS patients, further accumulation of these patients could clarify the association between advanced parental age at childbirth and epimutation in PWS patients.

Our study has some limitations. First, the number of patients with epi-IDs was obviously much lower $(20,000$ - fold) than that in the general population. Particularly, we included the limited number of patients with epi-IDs other than SRS and BWS. Because our subjects were patients referred to us for genetic testing for IDs, we did not include all patients with epi-IDs in Japan. Thus, we could not perform statistical tests for the comparison of the proportions of ART-conceived livebirths between patients with epi-IDs and the general population. These are inevitable limitations of our study, which focuses on rare diseases. Second, as in previous reports, we could not perform statistical analysis to determine whether epimutation is caused by ART or the confounding effect of advanced maternal childbearing age. Because mothers who received ART were mostly of advanced age in Japan, it is impossible to estimate the independent effects of ART itself. In addition, we could not evaluate whether the interaction between ART and maternal childbearing age induces an increase in epi-IDs. Third, the confounding effect of infertility was not evaluated, as we did not inquire about the reason for using ART in the questionnaire. Lastly, the effect of COS alone was not investigated, as we did not include COS into the ART procedure based on the JSOG's definition. A previous study revealed that COS interfered with maternal genomic imprinting in oocytes [7]. Further research is required to elucidate the effects of COS for the development of epi-IDs.

\section{Conclusions}

In summary, based on the prerequisite that most of the ART procedures in Japan are performed on mothers 

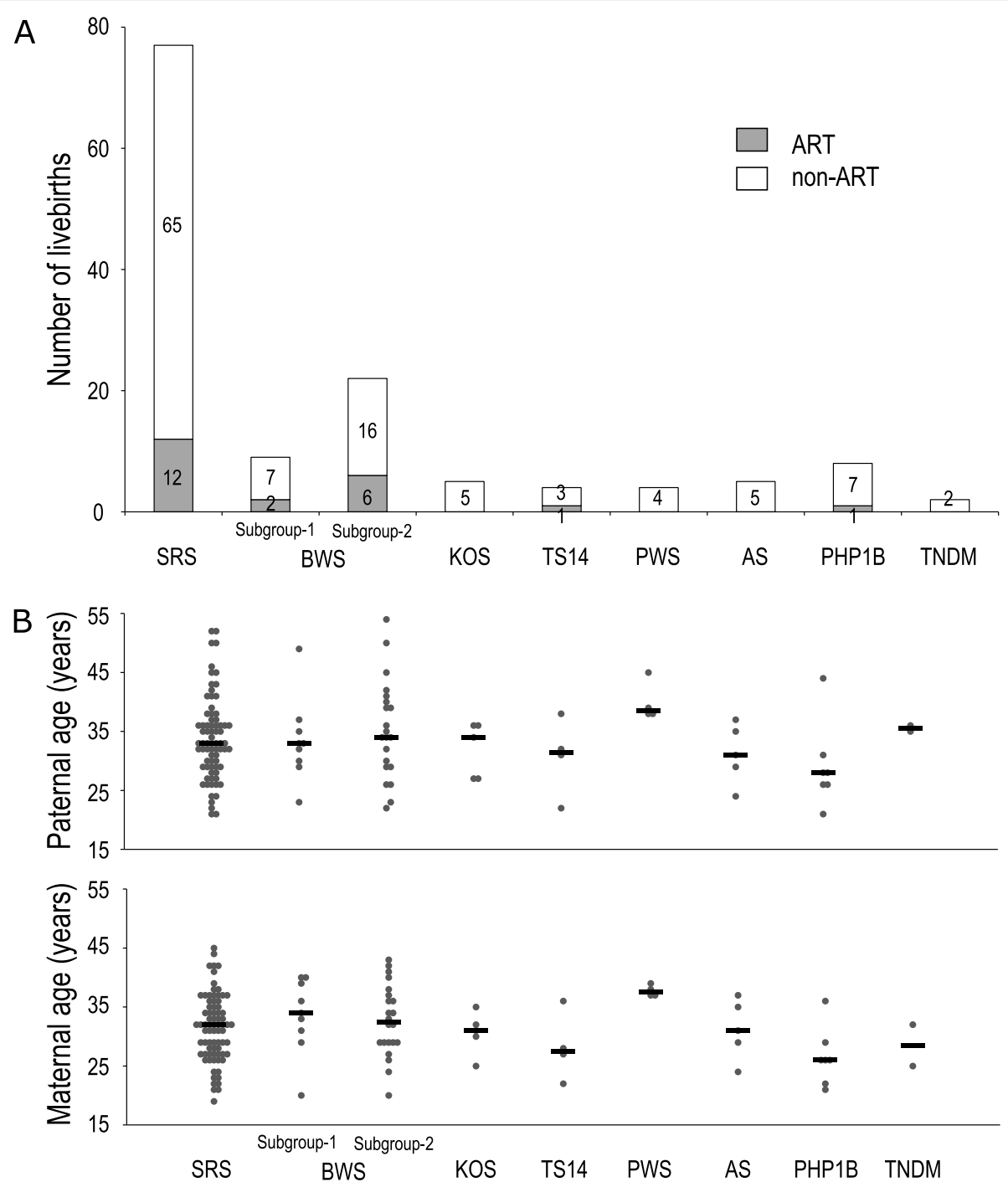

Fig. 4 Comparison across patients with eight epi-IDs. a The proportion of ART-conceived livebirths in patients with eight epi-IDs. Gray and white bars indicate the number of ART-conceived and non-ART-conceived livebirths in patients with each epi-ID, respectively. $\mathbf{b}$ The distribution of paternal and maternal ages at childbirth in patients with eight epi-IDs. Black bars indicate the median paternal or maternal age of patients with each epi-ID. ART, assisted reproductive technology; epi-IDs, epimutation-mediated imprinting disorders; SRS, Silver-Russell syndrome; BWS, Beckwith-Wiedemann syndrome; KOS, Kagami-Ogata syndrome; TS14, Temple syndrome; PWS, Prader-Willi syndrome; AS, Angelman syndrome; PHP1B, pseudohypoparathyroidism 1B; TNDM, transient neonatal diabetes mellitus

aged $\geq 30$ years, we concluded that ART can be a risk factor for the development of epi-IDs, especially SRS and BWS, for mothers aged $\geq 30$ years.

\section{Methods}

\section{Patients}

We enrolled 136 patients with epi-IDs confirmed by molecular studies including 21 previously reported patients (16 SRS patients, one KOS patient, and four TS14 patients) [22, 26-28]. Patients with seven IDs other than SRS were subjected to genetic testing. Regarding SRS, we included the patients with hypomethylation of the
H19/IGF2:IG-DMR who were suspected to have SRS due to SRS phenotype and/or severe growth failure. All patients were born from 1992 to 2017 and recruited from 2004 to 2019. Clinical information about parental age, conception (naturally or ART-conceived), and ART methods utilized in ART-conceived patients were obtained from the attending physicians by questionnaire. Because the JSOG has the database of the number of ARTconceived livebirths from IVF, ICSI, and FET, we classified patients conceived with IVF, ICSI, and FET as ARTconceived livebirths. Therefore, patients born after COS only were not included in ART-conceived livebirths. 
Table 2 Summary of the studies investigating the proportions of ART-conceived livebirths in imprinting disorders

\begin{tabular}{|c|c|c|c|c|c|c|c|}
\hline IDs & Country & $\begin{array}{l}\text { Number of } \\
\text { patients }\end{array}$ & $\begin{array}{l}\text { Number of patients } \\
\text { with molecular analysis }\end{array}$ & Non-ART & ART (\%) & $\begin{array}{l}\text { Genetic causes found } \\
\text { in ART-conceived IDs }\end{array}$ & $\begin{array}{l}\text { Interventions of } \\
\text { ART performed }\end{array}$ \\
\hline Sutcliffe, 2006* [18] & UK & & & & & & \\
\hline Total & & 317 & $12^{\mathrm{a}}$ & 295 & 22 & & $|V F| C S \mid$, \\
\hline BWS & & 79 & 8 & 68 & $11(13.9)$ & $\begin{array}{l}\text { epi, } 8^{\text {d }} \text {; not doing genetic } \\
\text { testing, } 3\end{array}$ & \\
\hline PWS & & 163 & 2 & 154 & $9(5.5)$ & $\begin{array}{l}\text { del, 2; not doing genetic } \\
\text { testing, } 7\end{array}$ & \\
\hline AS & & 75 & 2 & 73 & $2(2.7)$ & epi, $1 ;$ del, 1 & \\
\hline Doornbos 2007* [19] & Netherlands & & & & & & \\
\hline Total & & 220 & $11^{a}$ & 206 & 14 & & IVF, ICSI, \\
\hline BWS & & 71 & 6 & 65 & $6(8.5)$ & epi, $6^{d}$ & COS, IUI \\
\hline PWS & & 86 & 3 & 82 & $4(4.7)$ & $\begin{array}{l}\text { del, 3; not doing genetic } \\
\text { testing, } 1\end{array}$ & \\
\hline AS & & 63 & 2 & 59 & $4(6.3)$ & $\begin{array}{l}\text { del, } 2 \text {; not doing genetic } \\
\text { testing, } 2\end{array}$ & \\
\hline Tenorio 2016* [20] & Spain & & & & & & \\
\hline BWS & & 156 & $156^{\mathrm{b}}$ & 139 & $17(10.9)$ & epi, $15^{\text {d }}$; unknown, 2 & IVF, Al, COS \\
\hline Mussa, 2017* [21] & Italy & & & & & & \\
\hline BWS & & 38 & $7^{\mathrm{a}}$ & 31 & $7(18.4)$ & epi, $3^{\text {d}}$; UPD, 2; unknown, 2 & IVF, ICSI, IUI \\
\hline Hattori, 2019* [14] & Japan & & & & & & \\
\hline Total & & 931 & 556 & 888 & 43 & & IVF, ICSI \\
\hline SRS & & 67 & $22^{c}$ & 59 & $8(11.9)$ & epi, 5; not doing genetic testing, 3 & \\
\hline BWS & & 117 & 43 & 110 & $7(6.0)$ & epi, $4^{d}$; not doing genetic testing, 3 & \\
\hline PWS & & 520 & 366 & 496 & $24(4.6)$ & $\begin{array}{l}\text { epi, 6; del, 6; UPD, 9; not } \\
\text { doing genetic testing, } 3\end{array}$ & \\
\hline AS & & 227 & 147 & 223 & $4(1.8)$ & del, 4 & \\
\hline This study** & Japan & & & & & & \\
\hline Total & & 136 & $136^{\mathrm{b}}$ & 114 & 22 & & IVF, ICSI, FET \\
\hline SRS & & 77 & 77 & 65 & $12(15.6)$ & epi, 12 & \\
\hline BWS & & 31 & 31 & 23 & $8(25.8)$ & epi, $8^{\text {e }}$ & \\
\hline KOS & & 5 & 5 & 5 & 0 & & \\
\hline TS14 & & 4 & 4 & 3 & $1(25.0)$ & epi, 1 & \\
\hline PWS & & 4 & 4 & 4 & 0 & & \\
\hline AS & & 5 & 5 & 5 & 0 & & \\
\hline PHP1B & & 8 & 8 & 7 & $1(12.5)$ & epi, 1 & \\
\hline TNDM & & 2 & 2 & 2 & 0 & & \\
\hline
\end{tabular}

IDs imprinting disorders, ART assisted reproductive technology, BWS Beckwith-Wiedemann syndrome, PWS Prader-Willi syndrome, AS Angelman syndrome, SRS Silver-Russell syndrome, KOS Kagami-Ogata syndrome, TS14 Temple syndrome, PHP1B pseudohypoparathyroidism 1B, TNDM transient neonatal diabetes mellitus, UK United Kingdom, epi epimutation, del deletion, UPD uniparental disomy, IVF in vitro fertilization, ICSI intracytoplasmic sperm injection, COS controlled ovarian stimulation, IUI intrauterine insemination, $A I$ artificial insemination, FET frozen embryo transfer

*Patients with all genetic causes resulting in IDs were included

**Patients with only epimutation-mediated IDs were included

${ }^{a}$ Molecular analysis was performed in patients with ART-conceived IDs only

${ }^{\mathrm{b}}$ Molecular analysis was performed in all patients

Including 10 patients from our study

${ }^{\mathrm{d}}$ All patients had hypomethylated KCNQ10T1:TSS-DMR

e Patients had either hypomethylated KCNQ1OT1:TSS-DMR or hypermethylated H19/IGF2:IG-DMR

\section{Birth data of the Japanese general population}

The total number of livebirths and the distribution of maternal childbearing ages were obtained from the
Annual Nationwide Survey Data from the MHLW (http://www.mhlw.go.jp/toukei/list/81-1.html). This database showed the number of livebirths by five-year 
maternal age groups ( $\leq 14$ years, $15-19$ years, $20-24$ years, 25-29 years, 30-34 years, 35-39 years, 40-44 years, $45-49$ years, and $\geq 50$ years). Thus, we could not obtain the exact median age and 2.5th to 97.5th percentiles of the maternal childbearing ages of the general population from this database. In this regard, because median maternal childbearing age was included in the group of 25-29 years (1992-2002) and 30-34 years (2003-2017), we considered the approximate median maternal childbearing age as 27 years (1992-2002) and 32 years (2003-2017), respectively. Likewise, we considered the approximate 2.5 th percentile for childbearing age as 22 years and 97.5 th percentile for childbearing age as 37 years (1992-2007) and 42 years (2008-2017).

ART data, including the number of ART-conceived livebirths after ICSI (from 1985), IVF (from 1985), and FET (from 1989), and the age distribution of mothers who conceived with ART (from 2007) were obtained from an online registration system of the JSOG (https:// plaza.umin.ac.jp/ jsog-art/). This database includes data from almost all ART facilities and implemented ART cycles nationwide (604 facilities and 447,790 cycles in 2016) [2]. Detailed information collected from the registry has been reported previously [29].

\section{Comparison of the proportion of ART-conceived livebirths and parental ages}

We compared (1) the proportion of ART-conceived livebirths and (2) maternal childbearing ages in each year between patients with epi-IDs and the general population. The birth data of the general population was obtained from registry data of MHLW and JSOG as the control. Furthermore, we also compared the proportion of ART-conceived livebirths and parental ages among patients with eight epi-IDs.

\section{Molecular studies}

To detect patients with epimutation, we combined four genetic analysis as follows: (1) methylation analysis using pyrosequencing [28]; (2) methylation-specific multiplex ligation-dependent probe amplification (MS-MLPA, MRC Holland, Amsterdam, Netherlands) analysis utilizing commercially available MLPA probe mix for multiple segments on chromosomes 11 (ME030), 15 (ME028), and 20 (ME031); (3) microsatellite analysis for chromosomes 6 [30], 14 [31], 15 [16], and 20 [32] using patients' and their parental genomic DNA samples; and (4) an array-based comparative genomic hybridization $(\mathrm{aCGH})$ analysis using a custom-built array involving the imprinted regions related to IDs (Design ID 032112, Agilent Technologies, Santa Clara, CA, USA) [33]. The diagnostic process of each epi-ID is shown in Supplementary Figure S1.

\section{Statistical analysis}

For the comparison of the distribution of maternal childbearing ages between patients with epi-IDs and the general population, we used the median and 2.5th and 97.5th percentiles for continuous variables as summary statistics.

\section{Supplementary information}

Supplementary information accompanies this paper at https://doi.org/10. 1186/s13148-020-00900-x.

Additional file 1: Table S1. Frequency of epimutation in the eight representative imprinting disorders.

Additional file 2: Figure S1. The diagnostic process of eight epimutation-mediated imprinting disorders.

\section{Abbreviations}

aCGH: Array-based comparative genomic hybridization; ART: Assisted reproductive technology; AS: Angelman syndrome; BWS: Beckwith-

Wiedemann syndrome; CNVs: Copy number variants; COS: Controlled ovarian stimulation; DMRs: Differentially methylated regions; epi-IDs: Epimutationmediated imprinting disorders; FET: Frozen embryo transfer;

ICSI: Intracytoplasmic sperm injection; IDs: Imprinting disorders; IVF: In vitro fertilization; IVM: In vitro maturation; JSOG: Japan Society of Obstetrics and Gynecology; KOS: Kagami-Ogata syndrome; MHLW: Ministry of Health, Labor; MS-MLPA: Methylation-specific multiplex ligation-dependent probe amplification; PHP1B: Pseudohypoparathyroidism 1B; PWS: Prader-Willi syndrome; SRS: Silver-Russell syndrome; TNDM: Transient neonatal diabetes mellitus; TS14: Temple syndrome; UPD: Uniparental disomy

\section{Acknowledgements}

We are grateful to all patients and their parents for their cooperation. We thank Drs. Minoru Irahara and Akira Kuwahara for providing us with detailed ART data from JSOG. We thank Drs. Seiji Mizuno, Koji Muroya, Chikahiko Numakura, Sumito Dateki, Yutaka Nishimura, Shigeki Tanaka, Yasuhiro Naiki, Takahiro Okutani, Masataka Maeyama, Kenichi Kashimada, Keisuke Nagasaki, Masayo Yamazaki, Yukihiro Hasegawa, Tatsuya Watanabe, Sachiko Kitanaka, Seiji Sato, Tomonobu Hasegawa, Yasuyuki Fukuhara, Yuka Wada, Takao Togawa, Toshihiro Nagai, Nobuyuki Murakami, Hiroshi Suzumura, Shinji Saito, Motohide Goto, Shinichiro Sano, Yumiko Ikemoto, Katsutoshi Takahashi, Tomoki Kosho, Toru Yorifuji, Masako Aoki, Kenji Ihara, Yoko Miyoshi, Naoko Sato, Yuriko Katsushima, Junko Tsubaki, Hotaka Kamasaki, Junko Nishioka, Yuto Aoyagi, Masahito Yamamoto, Tsutomu Kamimaki, Katsumi Ushijima, Shuichi Yatsuga, Akira Motegi, Maiko Utoyama, Kazuki Yamazawa, Yasunori Wada, Ritsuko Furukawa, Shusuke Amakata, and Naoko Ishikawa for providing us with detailed clinical data and materials for molecular studies. We also thank Drs. Tomoko Fuke, Akie Nakamura, Takanobu Inoue, Sayaka Kawashima, and Mrs. Tamae Tanji for their support for molecular analysis.

\section{Authors' contributions}

KI performed the data analysis and wrote the paper. KM performed the molecular and data analysis. MM supervised the statistical analysis. TO recruited the cases enrolled in this study. TA and MF reviewed the paper and supervised the project. MK designed the project, wrote the paper, and gave the final approval of the version to be published. All authors read and approved the final manuscript.

\section{Funding}

This work was supported by grants from the National Center for Child Health and Development (28-6, 2019-B4), the Japan Agency for Medical Research and Development (AMED) (20ek0109373h0003), and Takeda Science Foundation.

Availability of data and materials

All data generated or analyzed during this study are available from the corresponding author on reasonable request. 


\section{Ethics approval and consent to participate}

This study was approved by the Institutional Review Board Committee at the National Center for Child Health and Development and performed after obtaining written informed consent to publish the clinical and molecular information.

\section{Consent for publication}

We obtained written informed consent from the patients or the patients' parents to publish patients' clinical and molecular information.

\section{Competing interests}

The authors declare that they have no competing interests.

\section{Author details}

'Department of Molecular Endocrinology, National Research Institute for Child Health and Development, 2-10-1 Okura, Setagaya-ku, Tokyo 157-8535, Japan. ${ }^{2}$ Department of Pediatrics, Keio University School of Medicine, 35 Shinanomachi, Shinjuku-ku, Tokyo 160-8582, Japan. ${ }^{3}$ Division of Biostatistics, Clinical Research Center, National Center for Child Health and Development, 2-10-1 Okura, Setagaya-ku, Tokyo 157-8535, Japan. ${ }^{4}$ Department of Informative Genetics, Environment and Genome Research Center, Tohoku University Graduate School of Medicine, 2-1 Seiryo-cho, Aoba-ku, Sendai 980-8575, Japan. ${ }^{5}$ Department of Pediatrics, Hamamatsu University School of Medicine, 1-20-1 Handayama, Higashi-ku, Hamamatsu 431-3192, Japan.

Received: 21 April 2020 Accepted: 8 July 2020

Published online: 22 July 2020

\section{References}

1. Adamson GD, de Mouzon J, Chambers GM, Zegers-Hochschild F, Mansour $\mathrm{R}$, Ishihara $\mathrm{O}$, et al. International Committee for Monitoring Assisted Reproductive Technology: world report on assisted reproductive technology, 2011. Fertil Steril. 2018;110:1067-80.

2. Ishihara O, Jwa SC, Kuwahara A, Ishikawa T, Kugu K, Sawa R, et al. Assisted reproductive technology in Japan: a summary report for 2016 by the Ethics Committee of the Japan Society of Obstetrics and Gynecology. Reprod Med Biol. 2019;18:7-16.

3. Canovas S, Ross PJ, Kelsey G, Coy P. DNA methylation in embryo development: epigenetic impact of ART (assisted reproductive technologies). Bioessays. 2017;39.

4. Hiura H, Okae H, Chiba H, Miyauchi N, Sato F, Sato A, et al. Imprinting methylation errors in ART. Reprod Med Biol. 2014;13:193-202.

5. Tucci V, Isles AR, Kelsey G, Ferguson-Smith AC, Erice Imprinting Group. Genomic imprinting and physiological processes in mammals. Cell. 2019; 176:952-65.

6. Monk D, Mackay DJG, Eggermann T, Maher ER, Riccio A. Genomic imprinting disorders: lessons on how genome, epigenome and environment interact. Nat Rev Genet. 2019;20:235-48.

7. Sato A, Otsu E, Negishi H, Utsunomiya T, Arima T. Aberrant DNA methylation of imprinted loci in superovulated oocytes. Hum Reprod. 2007;22:26-35.

8. Khoueiry R, Ibala-Rhomdane S, Mery L, Blachère T, Guérin JF, Lornage J, et al. Dynamic CpG methylation of the KCNQ1OT1 gene during maturation of human oocytes. J Med Genet. 2008:45:583-8.

9. White CR, Denomme MM, Tekpetey FR, Feyles V, Power SG, Mann MR. High frequency of imprinted methylation errors in human preimplantation embryos. Sci Rep. 2015;5:17311

10. Vincent RN, Gooding LD, Louie K, Chan Wong E, Ma S. Altered DNA methylation and expression of PLAGL1 in cord blood from assisted reproductive technology pregnancies compared with natural conceptions. Fertil Steril. 2016;106:739-48.e733

11. Nelissen EC, Dumoulin JC, Daunay A, Evers JL, Tost J, van Montfoort AP Placentas from pregnancies conceived by IVF/ICSI have a reduced DNA methylation level at the $\mathrm{H} 19$ and MEST differentially methylated regions. Hum Reprod. 2013;28:1117-26.

12. Santi D, De Vincentis S, Magnani E, Spaggiari G. Impairment of sperm DNA methylation in male infertility: a meta-analytic study. Andrology. 2017;5:695-703.

13. Adkins RM, Thomas F, Tylavsky FA, Krushkal J. Parental ages and levels of DNA methylation in the newborn are correlated. BMC Med Genet. 201 1;12:47.

14. Hattori H, Hiura H, Kitamura A, Miyauchi N, Kobayashi N, Takahashi S, et al. Association of four imprinting disorders and ART. Clin Epigenetics. 2019;11:21.
15. Cortessis VK, Azadian M, Buxbaum J, Sanogo F, Song AY, Sriprasert I, et al. Comprehensive meta-analysis reveals association between multiple imprinting disorders and conception by assisted reproductive technology. J Assist Reprod Genet. 2018:35:943-52.

16. Matsubara K, Murakami N, Nagai T, Ogata T. Maternal age effect on the development of Prader-Willi syndrome resulting from upd (15)mat through meiosis 1 errors. J Hum Genet. 2011;56:566-71.

17. Kindsfather AJ, Czekalski MA, Pressimone CA, Erisman MP, Mann MRW. Perturbations in imprinted methylation from assisted reproductive technologies but not advanced maternal age in mouse preimplantation embryos. Clin Epigenetics. 2019;11:162.

18. Sutcliffe AG, Peters CJ, Bowdin S, Temple K, Reardon W, Wilson L, et al. Assisted reproductive therapies and imprinting disorders -a preliminary British survey. Hum Reprod. 2006;21:1009-11.

19. Doornbos ME, Maas SM, McDonnell J, Vermeiden JP, Hennekam RC. Infertility, assisted reproduction technologies and imprinting disturbances: a Dutch study. Hum Reprod. 2007;22:2476-80

20. Tenorio J, Romanelli V, Martin-Trujillo A, Fernandez GM, Segovia M, Perandones $C$, et al. Clinical and molecular analyses of BeckwithWiedemann syndrome: comparison between spontaneous conception and assisted reproduction techniques. Am J Med Genet A. 2016;170:2740-9.

21. Mussa A, Molinatto C, Cerrato F, Palumbo O, Carella M, Baldassarre G, et al. Assisted reproductive techniques and risk of Beckwith-Wiedemann syndrome. Pediatrics. 2017;140:e20164311.

22. Kagami M, Nagasaki K, Kosaki R, Horikawa R, Naiki Y, Saitoh S, et al. Temple syndrome: comprehensive molecular and clinical findings in 32 Japanese patients. Genet Med. 2017;19:1356-66.

23. Goel NJ, Meyers LL, Frangos M. Pseudohypoparathyroidism type 1B in a patient conceived by in vitro fertilization: another imprinting disorder reported with assisted reproductive technology. J Assist Reprod Genet. 2018:35:975-9.

24. Fernandez M, Zambrano MJ, Riquelme J, Castiglioni C, Kottler ML, Juppner $\mathrm{H}$, et al. Pseudohypoparathyroidism type 1B associated with assisted reproductive technology. J Pediatr Endocrinol Metab. 2017:30:1125-32.

25. Potabattula R, Dittrich M, Bock J, Haertle L, Muller T, Hahn T, et al. Allelespecific methylation of imprinted genes in fetal cord blood is influenced by cis-acting genetic variants and parental factors. Epigenomics. 2018;10:1315-26.

26. Yamazawa K, Kagami M, Nagai T, Kondoh T, Onigata K, Maeyama K, et al. Molecular and clinical findings and their correlations in Silver-Russell syndrome: implications for a positive role of IGF2 in growth determination and differential imprinting regulation of the IGF2-H19 domain in bodies and placentas. J Mol Med (Berl). 2008;86:1171-81.

27. Fuke T, Mizuno S, Nagai T, Hasegawa T, Horikawa R, Miyoshi Y, et al. Molecular and clinical studies in 138 Japanese patients with Silver-Russell syndrome. PLoS One. 2013;8:e60105.

28. Kagami M, Kurosawa K, Miyazaki O, Ishino F, Matsuoka K, Ogata T. Comprehensive clinical studies in 34 patients with molecularly defined UPD(14)pat and related conditions (Kagami-Ogata syndrome). Eur J Hum Genet. 2015:23:1488-98.

29. Irahara M, Kuwahara A, Iwasa T, Ishikawa T, Ishihara O, Kugu K, et al. Assisted reproductive technology in Japan: a summary report of 1992-2014 by the Ethics Committee, Japan Society of Obstetrics and Gynecology. Reprod Med Biol. 2017:16:126-32

30. Yorifuji T, Matsubara K, Sakakibara A, Hashimoto Y, Kawakita R, Hosokawa Y, et al. Abnormalities in chromosome $6 q 24$ as a cause of early-onset, nonobese, non-autoimmune diabetes mellitus without history of neonatal diabetes. Diabetic medicine. 2015;32:963-7.

31. Kagami M, Nishimura G, Okuyama T, Hayashidani M, Takeuchi T, Tanaka S, et al. Segmental and full paternal isodisomy for chromosome 14 in three patients: narrowing the critical region and implication for the clinical features. Am J Med Genet A. 2005;138A:127-32.

32. Kawashima S, Nakamura A, Inoue T, Matsubara K, Horikawa R, Wakui K, et al. Maternal uniparental disomy for chromosome 20: physical and endocrinological characteristics of five patients. J Clin Endocrinol Metab. 2018;103:2083-8.

33. Kagami M, Kato F, Matsubara K, Sato T, Nishimura G, Ogata T. Relative frequency of underlying genetic causes for the development of UPD(14)pat-like phenotype. Eur J Hum Genet. 2012;20:928-32.

\section{Publisher's Note}

Springer Nature remains neutral with regard to jurisdictional claims in published maps and institutional affiliations. 